\title{
MITIGATION OF ICE LOADING ON OFF-SHORE WIND TURBINES FEASIBILITY STUDY OF A SEMI-ACTIVE SOLUTION
}

\author{
Arkadiusz Mróz ${ }^{*} \dagger$, Jan Holnicki-Szulc ${ }^{\dagger}$, and Tuomo Kärnä* \\ * Technical Research Centre of Finland (VTT) \\ Building and Transport \\ Vuorimiehentie 5, P.O.Box 2000, FI-02044 VTT, Finland \\ e-mail: tuomo.karna@vtt.fi, web page: http://www.vtt.fi \\ ${ }^{\dagger}$ Institute for Fundamental Technological Research (IPPT) \\ SMART-TECH Centre \\ ul. Swietokrzyska 21, 00-049 Warszwa, Poland \\ e-mail: amroz@ippt.gov.pl, holnicki@ippt.gov.pl, web page: http://www.ippt.gov.pl
}

Keywords: impact load mitigation, semi-active control, conical structures, ice loading

\begin{abstract}
This paper focuses on mitigation of dynamic loading induced on off-shore towers by drifting ice. Conical structures attached to off-shore towers at water level are widely used to reduce static and dynamic ice actions. The level of remaining forces is enough to cause severe tower vibrations and, in some situations also self induced excitation. A need for an additional device to reduce the remaining actions is recognized. Present study introduces a new, compliant connection between the cone and the tower. Parameters of the connection are controlled semi-actively to optimize the effect. Numerical studies are made to roughly estimate the effectiveness of the new solution. First, contact problem of ice sheet and cone is simulated using simplified material model for ice. Then a numerical model of a wind turbine tower is constructed. Dynamic interaction between cone and tower is simulated and the new solution effectiveness is discussed in terms of tower and blades displacements. Finally a laboratory experiment is proposed to further elaborate this numerical feasibility study.
\end{abstract}

\section{INTRODUCTION}

Many wind fields attractive for the wind industry (f.e. over Bothnian Bay) or large, unexploited oil fields are located off-shore, within Polar climate area, forcing some engineering structures like wind turbine towers, oil platform supports or lighthouses to 
withstand very harsh environmental conditions. Strong winds, sea wave loading, icing as well as drifting ice loading are among severe environmental actions, such structures must be resistant to. This paper focuses on mitigation of dynamic loading caused on off-shore towers by the drifting ice.

Ice fields often cover several square kilometers of sea and can drift with velocities of more than $1.0 \mathrm{~m} / \mathrm{s}$. Mechanical properties of ice such as bending strength, Young modulus, iso- or unisotrophy depend on several parameters, f.e. temperature, age of ice sheet or micro-structure of ice material. Due to its complexity an ice-sheet interacting with off-shore structure can produce a wide range of deformation states, each generating different reactions on the structure.

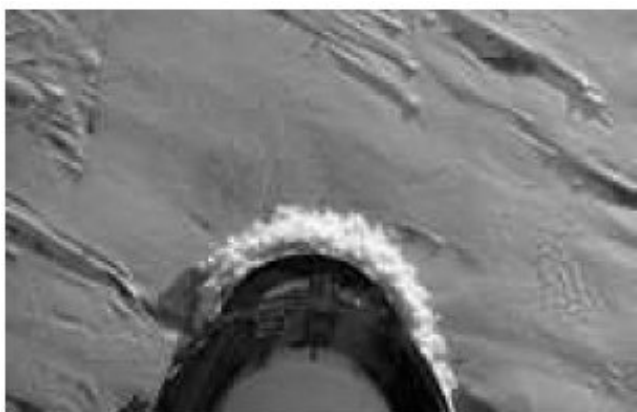

a)

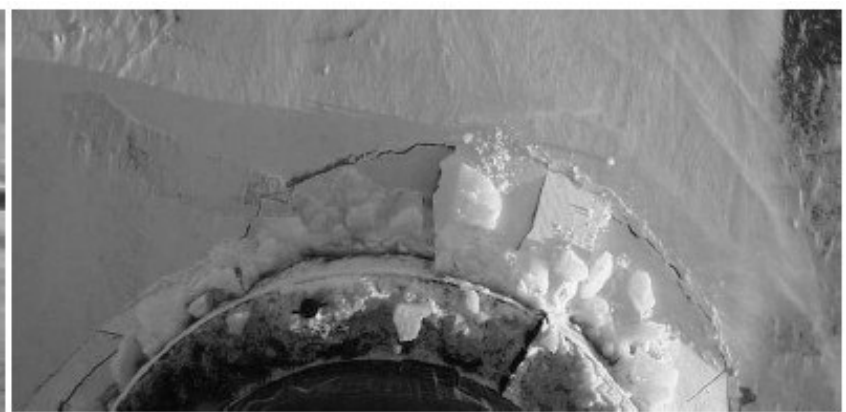

b)

Figure 1: Crushing (a) flexural (b) ice failure modes

Depending on the mechanical properties of ice, its thickness, drift velocity, as well as geometry of the structure, two modes of ice failure have been identified: crushing mode and bending mode. Their camera images $^{1}$ are shown on Figure 1, whereas the corresponding reaction forces ${ }^{2}$ are shown on Figure 2. In practice a mixture of these two failure modes is very often present. Time period between consecutive peaks on Figure 2a) depends on the flow velocity and breaking length of the ice sheet. Breaking length is defined as the distance between the zone of ice edge/structure contact and the first circumferential crack ${ }^{3}$. If the breaking length divided by the ice flow velocity equals to the fundamental own frequency of the tower then a narrow band excitation may occur in the structure.

For cylindrical structures interacting with drifting ice (of thickness $0.2 \mathrm{~m}$ and more) the most probable ice failure mode is crushing ${ }^{1}$. Such actions could generate as high dynamic forces as $5 \mathrm{MN}$ and were of critical concern for the structural designers.

A successful meassure to reduce ice induced dynamic forces was the introduction of conical structures fixed to towers at the water level (Figure 3). Their aim is to promote the bending failure of an ice sheet by means of introducing a vertical component to the total interaction force ${ }^{4}$. 
A. Barker et al. / Cold Regions Science and Technology 41 (2005) 1-23

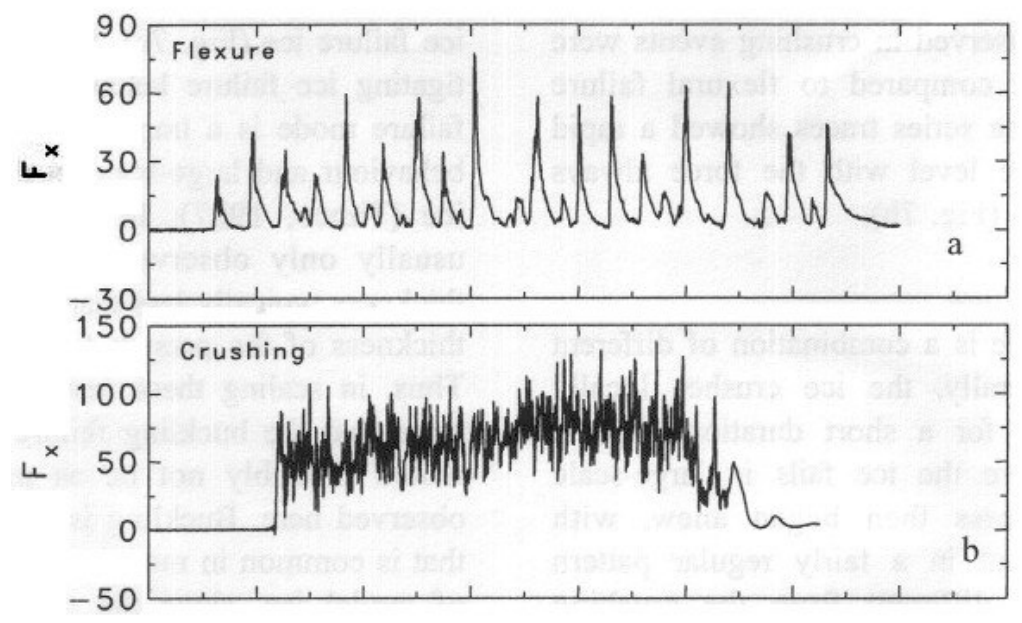

Figure 2: Typical measured ice forces acting on tower

Since the total interaction force acts always normal to the surface of structure, the slope of a cone introduces the vertical component. When the value of vertical force exceeds a certain level, the ice bending strength is reached and the bending failure occurs in the ice sheet. The horizontal force corresponding to the failure point, i.e. the maximum force inducing tower vibrations is substantially decreased compared to the case without a cone, but can still reach as high values as $800 \mathrm{kN}$.
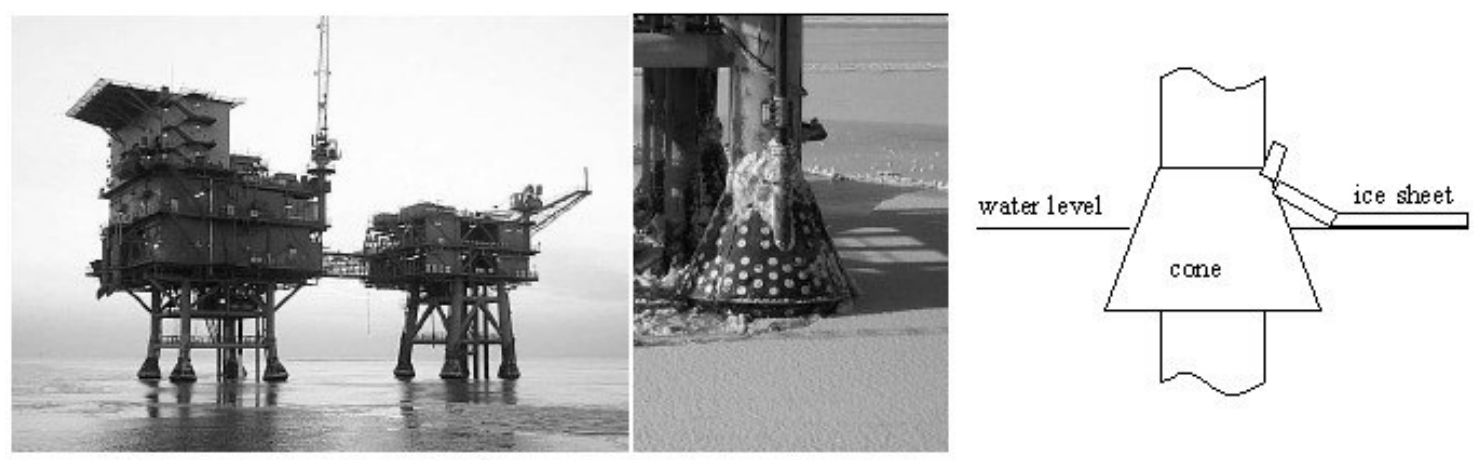

Figure 3: Conical structures attached to oil platform

Therefore a further need to mitigate the response of the structure and/or to reduce the ice induced loading is recognized. Prospects of using either passive or semi-active tuned mass dampers were discussed by Karna and Kolari ${ }^{5}$. Approaches to use a compliant connection instead of fixed cone were investigated by Lindqvist and Juurmaa ${ }^{6}$, and Wang ${ }^{7}$ In this paper a numerical study of another semi-active solution is made, aiming at mitigation of drifting ice induced forces. 


\section{IDEA OF SMART CONE}

The idea of Smart Cone is to further decrease the horizontal component of ice loading which causes tower vibrations, while preserving the vertical force which causes the bending failure of ice sheet. This could be achieved by introducing a new cone-tower interface, as shown on Figure 4.

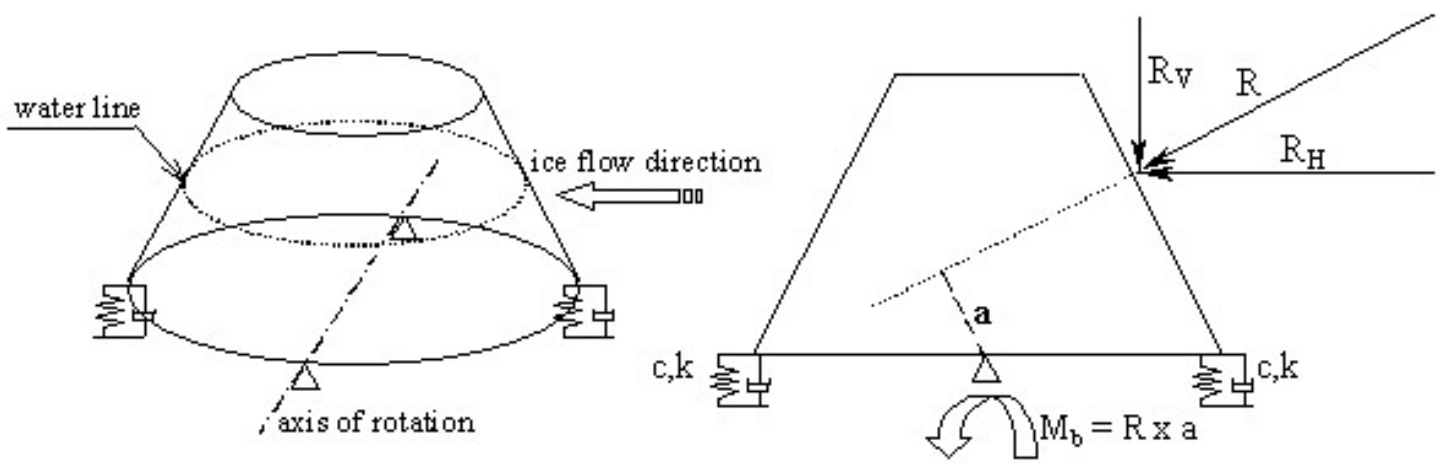

Figure 4: Compliant connection between cone and tower

Present, fixed connection between cone and the tower is replaced with a compliant connection based on a set of springs and dampers. Furthermore translation is constrained along the axis perpendicular to the ice flow direction. As long as the eccentricity "a" is non-zero, cone has the ability to rotate about this axis. This results in new behavior of the cone during a cone-ice contact event and consequently leads to different stress distribution within the ice sheet. In turn also cone-ice interaction forces and loading of the tower are changed. The effectiveness of present solution is dependent on the water level, as eccentricity "a" decreases with the decrease of water level. In more detail the principle of Smart Cone behavior can be described as follows. Since the cone-tower connection is compliant, the compressive stresses generated in the ice sheet by ice pressing against the cone are limited. In turn the horizontal component of the total interaction force is substantially reduced. The more compliant connection i.e. smaller values of parameters $\mathrm{c}$ and $\mathrm{k}$, the bigger decrease in horizontal reaction should be obtained. On the other hand rotation of the cone causes up-lifting of the ice sheet and thus its bending. This allows for the bending failure of the ice sheet.

Between ice failure and the instant of next cone-ice contact, the cone should return to its initial position. For this purpose high values of parameters $\mathrm{c}$ and $\mathrm{k}$ are preferred. Small values of these parameters from effectiveness standpoint and high values for above mentioned reason constitute the principle of the control strategy for the semi-active adaptation of spring and damper coefficients. 


\section{NUMERICAL SIMULATIONS - INTRODUCTION}

A complex ice material mechanical model capable of simulating bending and crushing failure modes is currently under development. Lack of such material model forbids a full icecone-tower dynamic simulation, where ice loading is modeled by periodically changing contact forces between ice sheet and the cone. In order to assess the efficiency of present solution the task has been split into two simulations carried out in ABAQUS/Standard FEM software.

I. Static analysis of cone-ice interaction. The aim of this simulation is to compare the reaction forces generated for the new solution with the reference case, for the same value of displacement prescribed to the ice sheet. A simplified elastic-plastic model is used for the ice material.

II. Dynamic analysis of the wind turbine tower. Static forces from Simulation I. are used to generate periodic loading pattern. The aim of this simulation is to discuss the effectiveness of the new solution in terms of top of tower and tip of blade displacements. Control strategy is implemented to control the cone behavior. Inertia effect of the swinging cone on the behavior of tower is briefly analyzed.

\section{SIMULATION OF THE CONE-ICE INTERACTION}

\subsection{FEM model}

The general set-up for the simulation is shown on Figure 5. The ice sheet was modeled using simple elastic-plastic material model with 4-node shell elements. Boundary conditions for the ice field were applied relatively far from the cone-ice contact area. The cone was modeled with 4-node rigid body elements. Cone rotation about Axis 3 was controlled by Axial Connector elements with elasticity and damping behavior defined. Note that velocities and thus also viscous forces can be calculated in static analysis. Water damping is discussed in 5.5.2. It is assumed that the water damping is included in the a.m. connector damping property.

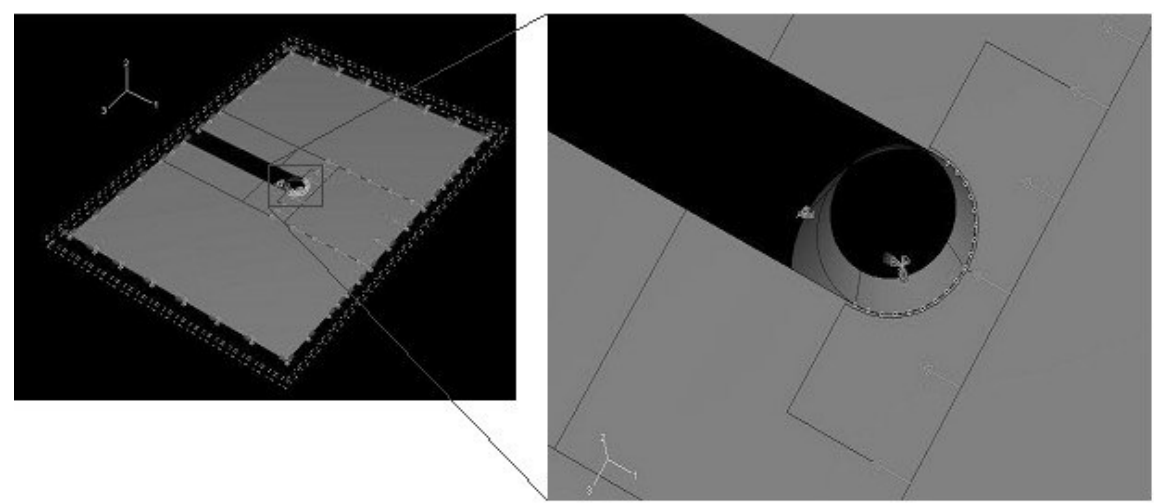

Figure 5: FEM model general view 
A contact problem was defined between the cone and edge of the ice sheet. Tangential contact behavior was assumed as static friction with friction coefficient $\mu=0.15$.

The total displacement of $0.05 \mathrm{~m}$ was applied gradually in 200 increments over a time period of $0.05 \mathrm{sec}$. This corresponds to the ice flow velocity of $1.0 \mathrm{~m} / \mathrm{s}$. A General Static step was created to perform the analysis.

For the reference case the same model was used, except all d.o.f.'s were constrained for the cone, including rotation about Axis 3.

\subsection{Results of simulation}

Results of the simulation are discussed in terms of (Figure 6):

a) reaction forces at the cone reference point;

b) stresses in the ice sheet, where the initial crack would occur;

c) horizontal displacement of the top edge of the cone.
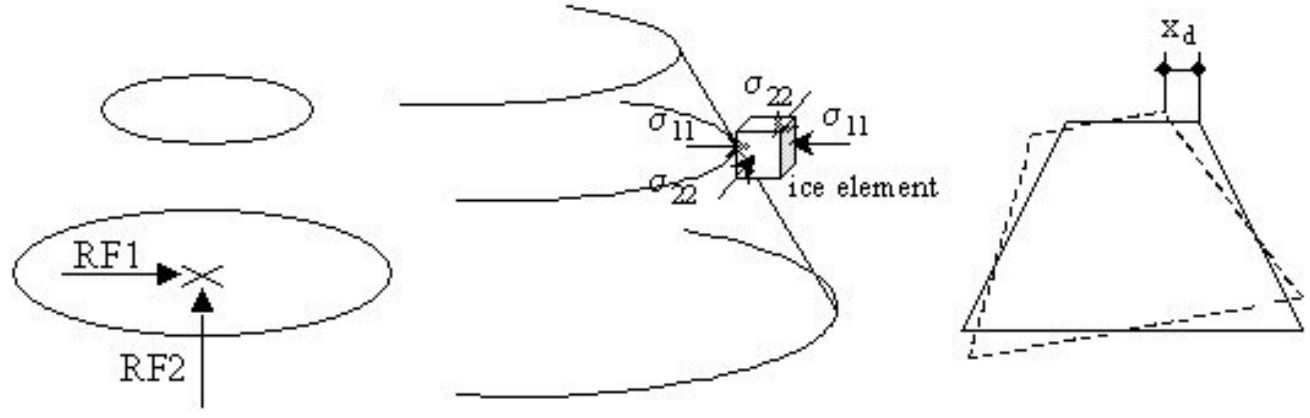

Figure 6: Chosen result quantities

\begin{tabular}{|c|c|c|c|c|c|c|c|}
\hline \multirow[b]{2}{*}{ Description } & \multicolumn{2}{|c|}{ Parameters } & \multirow[b]{2}{*}{$\mathrm{RF} 1[\mathrm{kN}]$} & \multirow{2}{*}{$\mathrm{RF} 2[\mathrm{kN}]$} & \multirow[b]{2}{*}{$\sigma_{11}[\mathrm{kPa}] *$} & \multirow[b]{2}{*}{$\sigma_{22}[\mathrm{kPa}] *$} & \multirow[b]{2}{*}{$\mathrm{x}_{\mathrm{d}}[\mathrm{cm}]$} \\
\hline & $\begin{array}{l}\text { Spring stiff. } \\
\mathrm{k}[\mathrm{N} / \mathrm{m}]\end{array}$ & $\begin{array}{l}\text { Ice thick. } \\
\mathrm{t}[\mathrm{m}]\end{array}$ & & & & & \\
\hline Ref. case, cone fixed & - & 0.50 & 322.8 & 128.1 & -458.7 & 152.7 & - \\
\hline New solution & 50000 & 0.50 & 190.2 & 111.4 & -213.2 & 422.6 & 7.05 \\
\hline New solution & 100000 & 0.50 & 206.6 & 111.6 & -240.2 & 399.6 & 6.91 \\
\hline New solution & 250000 & 0.50 & 252.0 & 111.8 & -316.3 & 331.1 & 6.50 \\
\hline New solution & 500000 & 0.50 & 278.9 & 116.3 & -368.7 & 268.7 & 4.59 \\
\hline Ref. case, cone fixed & - & 0.75 & 890.7 & 360.1 & -506.2 & 231.0 & - \\
\hline New solution & 50000 & 0.75 & 463.6 & 324.9 & -238.5 & 490.1 & 7.50 \\
\hline New solution & 100000 & 0.75 & 481.6 & 325.3 & -250.3 & 483.3 & 7.43 \\
\hline New solution & 250000 & 0.75 & 533.6 & 325.9 & -289.2 & 454.0 & 7.26 \\
\hline New solution & 500000 & 0.75 & 615.1 & 326.2 & -347.9 & 408.9 & 7.01 \\
\hline
\end{tabular}

* (+) - tension, (-) - compression

Table 1: Results comparison 
For both considered ice thickness' the best results were obtained for the lowest considered spring stiffness. If there was no spring at all then the RF1 reduction would be even more significant but the device could not return to its initial position. It can be seen from Table 1 that for $\mathrm{k}=50 \mathrm{kN} / \mathrm{m}$ there is still $41 \%$ and $48 \%$ mitigation of the horizontal force, for ice sheet thickness 50 and $75 \mathrm{~cm}$ respectively. The vertical force is not affected significantly by the new solution as values of RF2 are decreased only by $13 \%$ and $10 \%$ respectively. In practice the ice bending would not be affected allowing for the bending failure of the ice sheet.

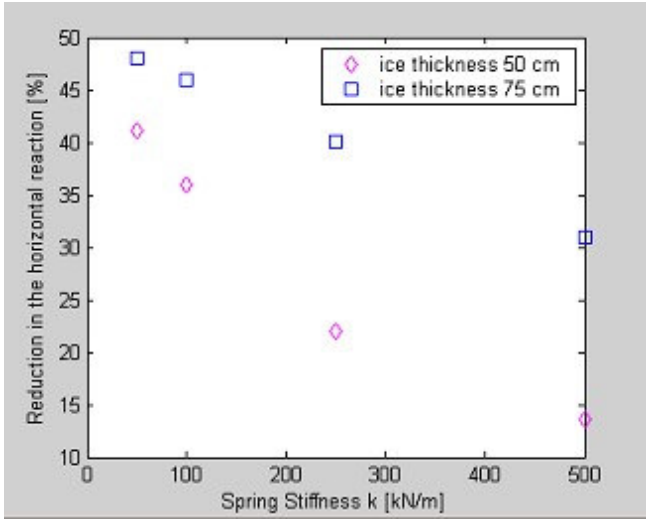

Figure 7: Horizontal reaction change as function of spring stiffness

The dependency of the horizontal reaction mitigation on the spring stiffness is shown on Figure 7.
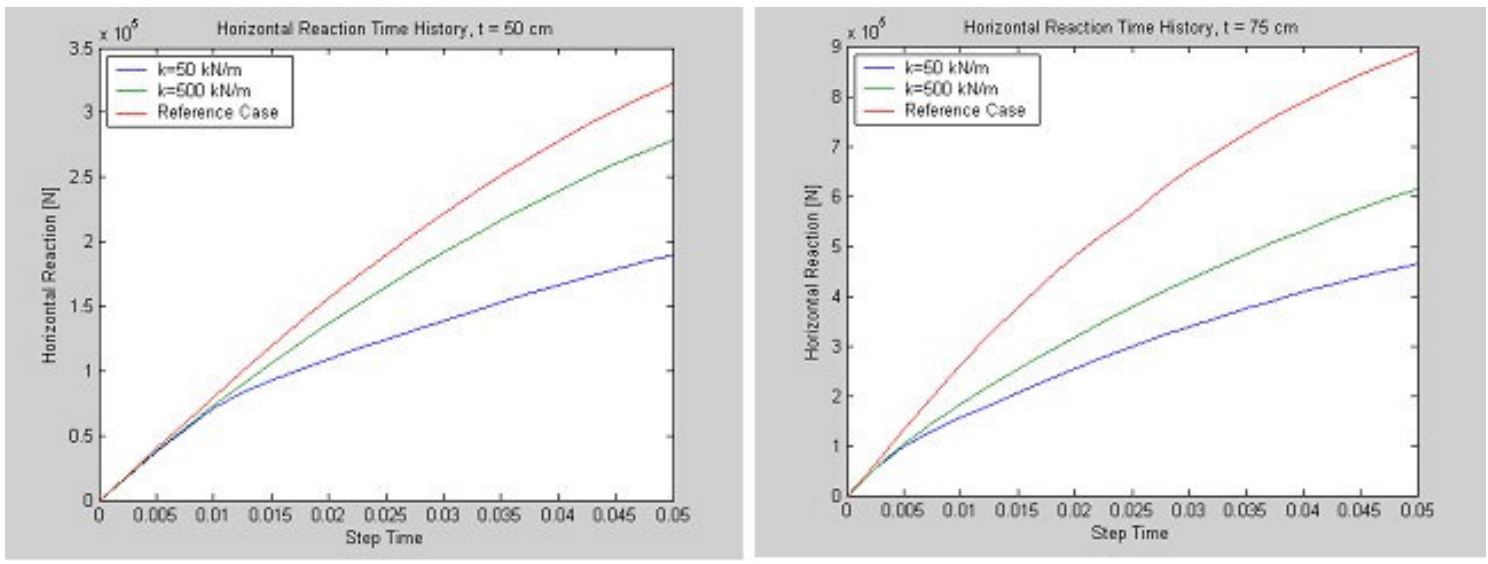

Figure 8: Time history of horizontal reaction change 
Figure 8 represents the Time History data showing the reaction increase with the increase in prescribed displacement. Non-linear growth is implied by the early yielding in the ice sheet.

It can also be seen in Table 1 that compressive stresses $\sigma_{11}$ which are generated by the ice pressing against the cone and thus produce the horizontal reaction force, are decreased by $54 \%$ and $53 \%$ for considered ice thickness'. In turn, stresses $\sigma_{22}$ which are tensile and in practice would result in a radial crack are clearly increased. It can be hoped that the new solution would produce a clearer bending failure in the ice sheet.

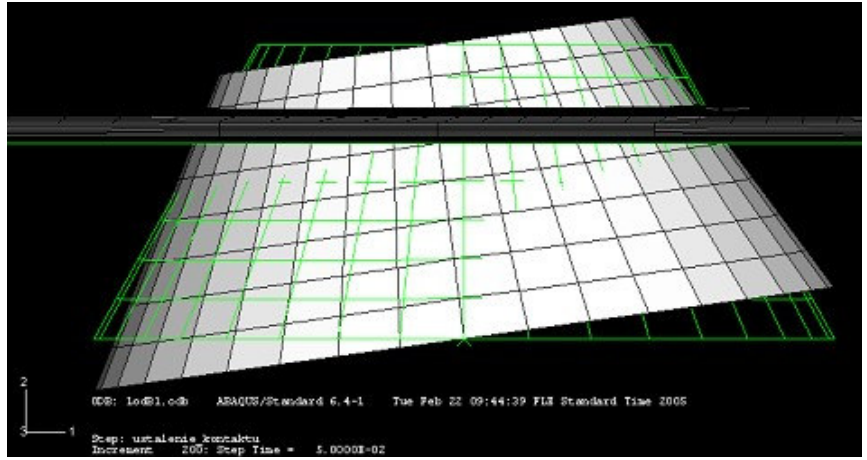

Figure 9: Deformed model

Figure 9 illustrates the final configuration of the cone and bending of the ice sheet (connector elements not displayed).

The initial full contact between cone surface and the ice sheet edge is reduced to about $30 \%$ due to the geometry incompliance after deformation.

The cone edge displacement $\mathrm{x}_{\mathrm{d}}$ is less than $10 \mathrm{~cm}$ in all cases, which is reasonable from the technological constrains point of view. It is finally worth mentioning that a decrease in the spring stiffness does not cause a significant increase in $x_{d}$. It is due to the fact that rotation of cone is constrained not only by springs but also by the ice sheet itself.

\subsection{Conclusions}

The simulation shows that the proposed solution could reduce the horizontal reaction by $41 \%$ and $48 \%$ for considered ice thickness'. Spring and damper coefficients should be possibly small from the effectiveness standpoint, allowing for the cone rotation.

\section{SIMULATION OF THE WIND TURBINE TOWER}

\subsection{FEM model in ABAQUS/Standard}

Main features of the wind turbine structure dynamic model are shown on Figure 10. Turbine mass elements and blades were connected to the tower with rigid connector elements in order to obtain a realistic mass distribution in space. The additional water 
mass effect, discussed in 5.5.1, is included in the model. The total weight of the structure is $186782+3 \times 7006+141020=348020 \mathrm{~kg}$.
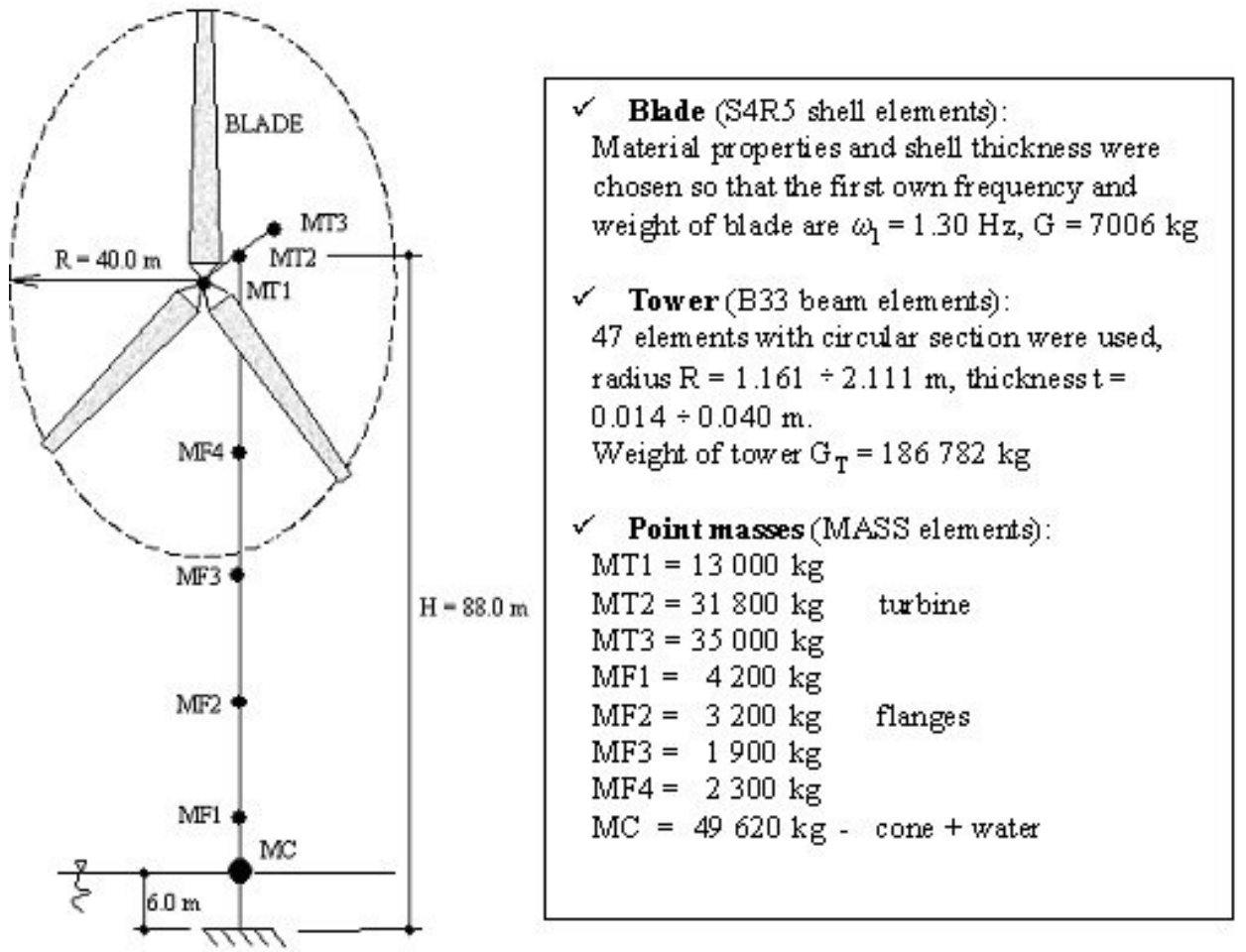

Figure 10: Dynamic model of the wind turbine tower

For the reference case the cone was modeled as a point mass MC. For the new solution simulation the cone was modeled with shell elements, and had the same mass as the point mass MC.

\subsection{Own frequencies extraction}

Table 2 lists first 7 own frequencies of the structure.

\begin{tabular}{|c|c|c|}
\hline Mode & Frequecy $[\mathrm{Hz}]$ & Period $[\mathrm{sec}]$ \\
\hline 1 & 0.320 & 3.125 \\
\hline 2 & 0.322 & 3.106 \\
3 & 0.985 & 1.015 \\
\hline 4 & 1.129 & 0.886 \\
\hline 5 & 1.293 & 0.773 \\
\hline 6 & 1.557 & 0.642 \\
\hline 7 & 2.230 & 0.448 \\
\hline
\end{tabular}

Table 2: Own frequencies of wind turbine tower 
The fundamental frequency of the whole structure is much lower $(0.32 \mathrm{~Hz})$ than the first own frequency of a blade $(1.30 \mathrm{~Hz})$. Free vibrations simulation shows that blades vibrate with higher frequency than the tower.

\subsection{Damping}

The structural damping was assumed to be $0.5 \%$ of the critical damping. Rayleigh mass and stiffness proportional damping coefficients were obtained from equation (1).

$$
\xi_{i}=\frac{\alpha_{R}}{2 \cdot \omega_{i}}+\frac{\beta_{R} \cdot \omega_{i}}{2}
$$

$\xi$ i was assumed $0.5 \%$ for $\omega_{1}=0.32 \mathrm{~Hz}$ and $\omega_{7}=2.23 \mathrm{~Hz}$, which gives damping coefficients of $\alpha_{R}=0.0028$ and $\beta_{R}=0.0039$.

\subsection{Loading}

\subsubsection{Critical breaking length}

If the ice loading period, i.e. breaking length divided by flow velocity happens to be close to the first own period of the tower, then narrow band excitation may be induced. Assuming the flow velocity equal to $1.0 \mathrm{~m} / \mathrm{s}$, the critical breaking length can be found:

$$
L_{b}=\frac{1}{0.32 \mathrm{~Hz}} \cdot 1.0 \frac{\mathrm{m}}{\mathrm{s}}=3.13 \mathrm{~m}
$$

From works by $\mathrm{Li}$ and $\mathrm{Yue}^{8}$ and $\mathrm{Li}$ et $\mathrm{al}^{3}$ the ice thickness corresponding to this critical breaking length can be obtained from the formula:

$$
L_{b}=k \cdot \xi^{\frac{1}{3}} \cdot h
$$

where $\mathrm{k}$ depends on cone geometry and ice-cone contact parameters, and $\xi$ depends on friction coefficient and inclination angle of the cone.

For the contact extent of $30 \%$ of the initial contact, friction coefficient $\mu=0.15$, water diameter of cone $\mathrm{D}=4.80 \mathrm{~m}$, and cone inclination angle $\alpha=60^{\circ}$, it follows that the obtained in (2) critical breaking length corresponds to ice thickness of ca. $50 \mathrm{~cm}$. Therefore results of Simulation I obtained for ice thickness $50 \mathrm{~cm}$ were chosen for amplitudes of ice loading, with period of loading equal to $\mathrm{T}_{1}=3.13 \mathrm{sec}$.

\subsubsection{Reference case loading}

In the reference case of the tower with fixed cone, the cone was modeled as a point mass and rigid, weightless cantilevers were added to properly apply the ice forces (Figure 11). 


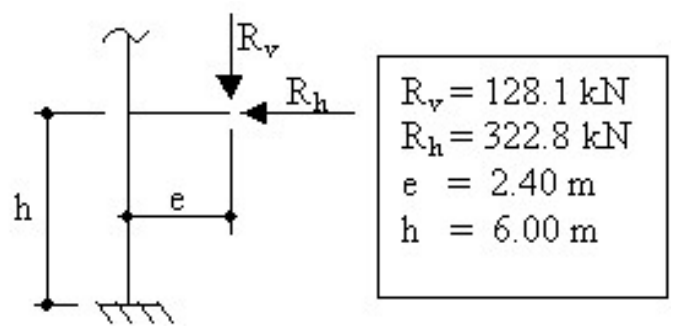

Figure 11: Reference case loading

For the loading amplitudes refer to Figure 13 a).

\subsubsection{New solution loading}

In the new solution case of compliant cone-tower connection, the ice forces were applied directly to the tower, which corresponds to the reaction forces at the cone reference point in Simulation I. The cone displacement was prescribed in order to simulate the behavior of moving cone and its influence on the tower, and to obtain spring and damper forces (Figure 12).

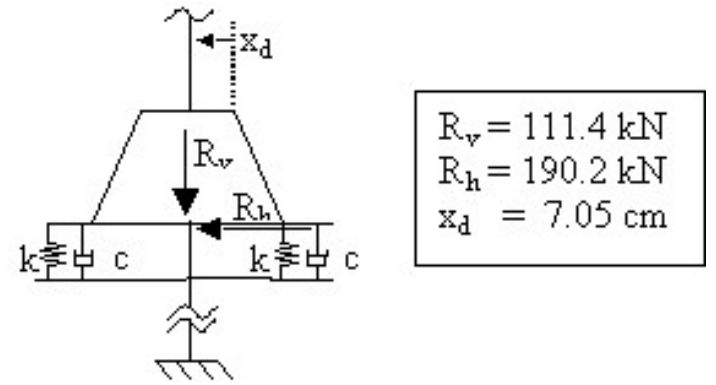

Figure 12: New solution loading

For the loading amplitudes refer to Figure 13 a) and b).

\subsubsection{Amplitudes}

Ice forces amplitude shape was assumed according to $\mathrm{Qu}$ at al. ${ }^{9}$. Period of loading is $3.13 \mathrm{sec}$ and corresponds to the own frequency of the tower. Value between peaks is assumed zero, which is realistic for narrow structures, where almost none of the broken ice stays on the cone. It is also assumed that the proposed solution will not affect the period of excitation.

The prescribed displacement is ramped from zero to its maximum value and then released so that the cone can move between peaks. 


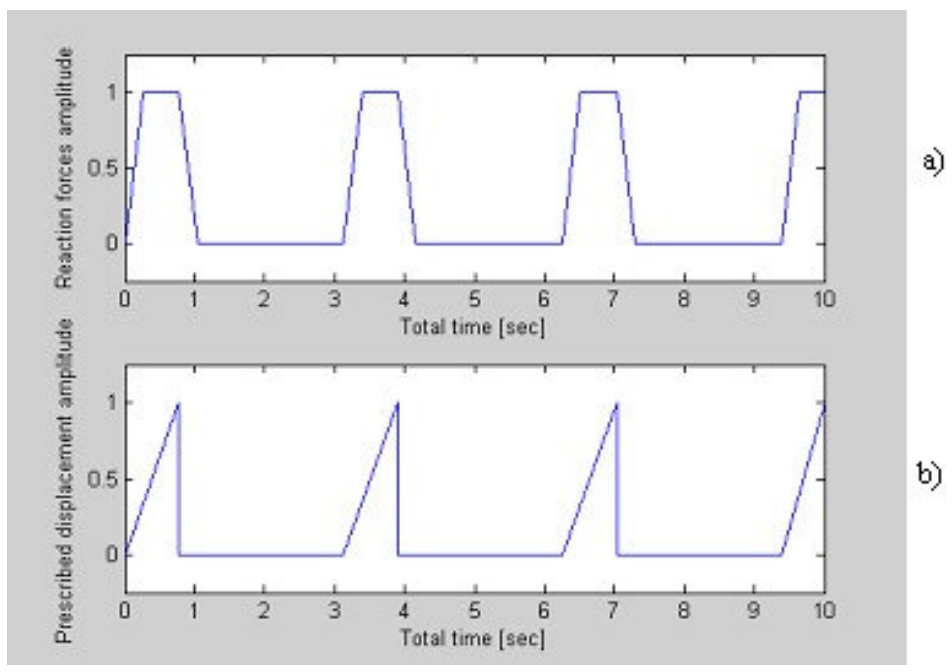

Figure 13: Ice force (a) and prescribed displacement (b) amplitudes

\subsection{The cone in new solution simulation}

\subsubsection{Cone structure}

The cone was modeled with 4-node shell elements of thickness $24 \mathrm{~cm}$ and average density corresponding to $20 \mathrm{~cm}$ concrete $+20 \mathrm{~mm}$ steel lining on the inside and outside. This gives the mass of cone equal to $40880 \mathrm{kgs}$.

For the submerged part additional mass was included in the cone weight equal to the mass of water displaced by the cone. Assuming that the inside of the cone is filled with water, the volume of water displaced is $36.44 \mathrm{~m}^{2} \times 0.24 \mathrm{~m}=8.745 \mathrm{~m}^{3}$. This gives the additional mass of $8745 \mathrm{kgs}$ and the total mass of cone equal to $40880+8745=49625$ kgs.

\subsubsection{Water damping effect}

According to J.F.Wilson ${ }^{10}$ the damping force characterizing the fluid-structure interactions can be calculated from the formula:

$$
F_{d}=\left(\hat{c}+C^{\prime}{ }_{d} \cdot \rho \cdot \frac{D}{2}\right) \cdot v
$$

where:

$\hat{c} \quad$ - structural damping coefficient

$\rho$ - fliud density

$C^{\prime}{ }_{d}$ - modified drag coefficient

D - water diameter of the structure 
Assuming that the structural damping is already taken into account in material properties, and after calculating the modified drag coefficient, the water-structure interaction damping coefficient can be obtained as $1505[\mathrm{~N} /(\mathrm{m} / \mathrm{s})]$.

\subsection{Control}

\subsubsection{Cone-tower interface}

The static model shown on Figure 4 was realized by means of connector elements tied to rigid cantilevers acting as a cone base. Join Type connector elements were used for translation constraints modeling the axis of rotation. Axial Type connector elements with stiffness and damping behavior were used to model springs and dampers. General view of the cone-tower connection is shown on Figure 14.

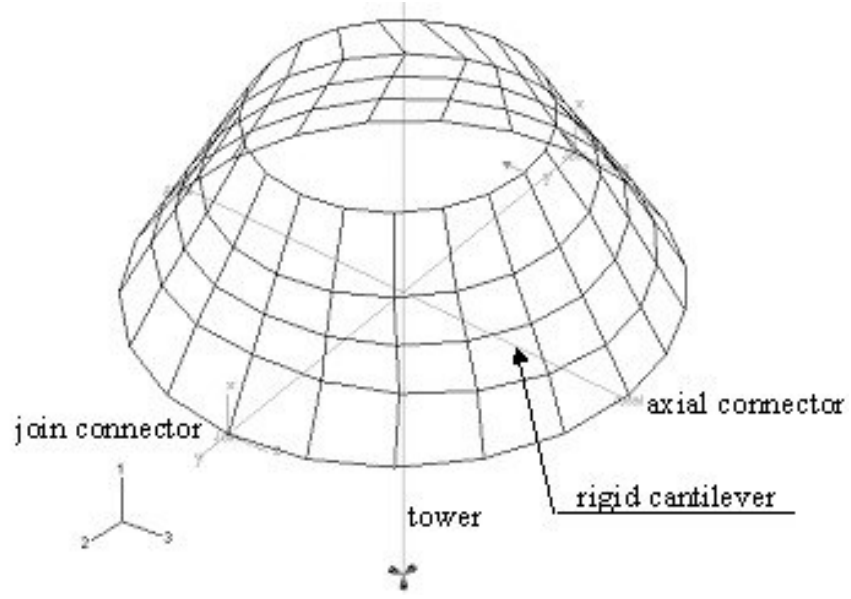

Figure 14: Cone - tower connection

\subsubsection{Stiffness and damping control}

Various values of spring and damper coefficients were considered. The goal was to obtain such values that the effectiveness of the solution is high - on one hand, and the cone is allowed to return to its initial position before the next peak - on the other. The general conclusion was that for higher values of spring and damper coefficients the cone returns to its initial position, but the effectiveness of the solution drops as can be seen on Figure 7. On the other hand for small values the effectiveness is the highest, but the cone movement is not damped out before the next peak. Furthermore the water damping is not enough to damp out the cone movement.

Because of the above the adaptation of mentioned parameters is necessary in order to obtain the desired effect. During the ice-cone contact event the cone-tower connection should be as compliant as possible to optimize the effect. Then, in between peaks the compliance should be adapted allowing for the returning of cone. 
It was assumed that the spring stiffness and the damping coefficients can be adjusted by one order of magnitude. The following procedure controls the real time adaptation of these parameters during the numerical analysis:

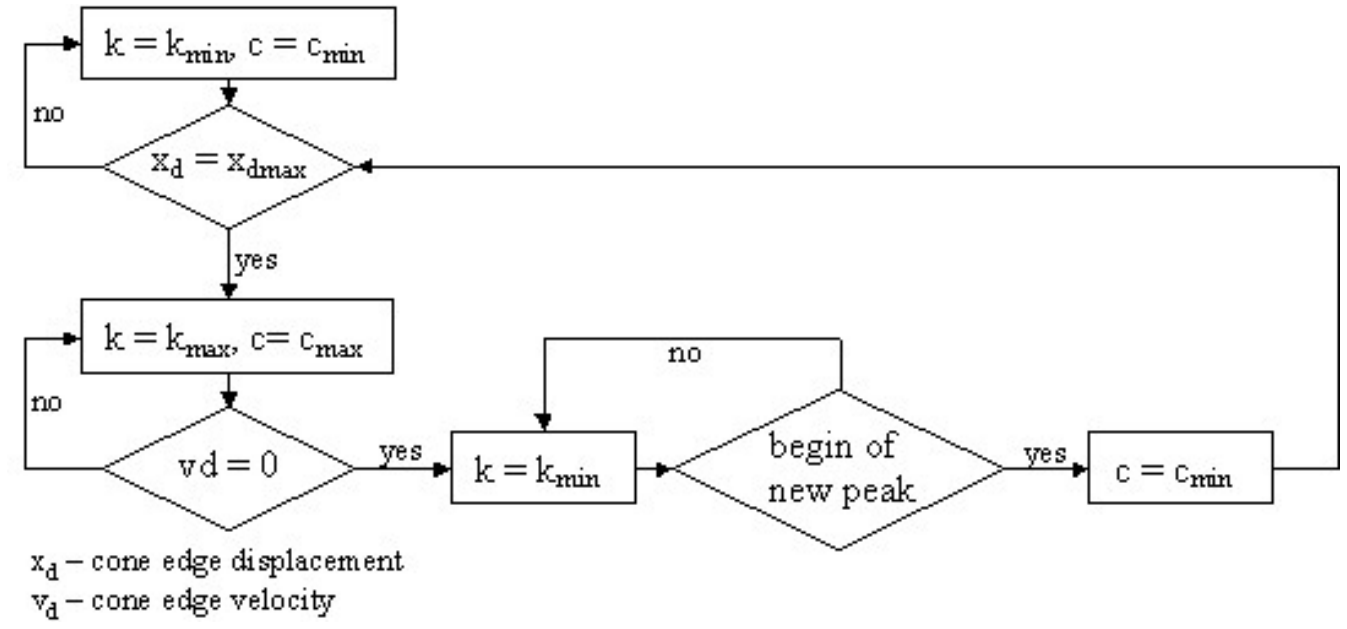

Figure 15: Control procedure

Stiffness and damping parameters were updated instantly and their values were:

$$
\begin{array}{ll}
\mathrm{k}_{\max }=500 \mathrm{kN} / \mathrm{m} & \mathrm{k}_{\min }=50 \mathrm{kN} / \mathrm{m} \\
\mathrm{c}_{\max }=40 \mathrm{kN} /(\mathrm{m} / \mathrm{s}) & \mathrm{c}_{\min }=4 \mathrm{kN} /(\mathrm{m} / \mathrm{s})
\end{array}
$$

Up to the point where both the loading and prescribed displacement begin to decrease the control parameters have their minimum values, which corresponds to conditions assumed in Simulation $I$.

In order to implement the control procedure in ABAQUS/Standard two FORTRAN procedures were used. UFIELD procedure updates values of field variables which in turn control the spring and damper behavior parameters. URDFIL procedure defines and updates readings from displacement, velocity and load sensors.

\subsubsection{Dependence of axis of rotation on the ice flow direction}

In practice the ice field flow changes direction and therefore the structure may come to contact with ice sheet at any point around its circumference. Because of this, it is also a task for the control device to adjust the axis of cone rotation to be always perpendicular to the ice flow direction. This could be realized by means of some structural fuse type of device that either imposes or releases constrains in some point.

This feature is not included in the present work, as the ice flow direction is assumed to be perpendicular to the axis of cone rotation. 


\subsection{Results of simulations}

\subsubsection{Wind turbine response to the dynamic loading}

On Figure 16 horizontal displacements of top of tower and tip of blade are compared with the reference case.

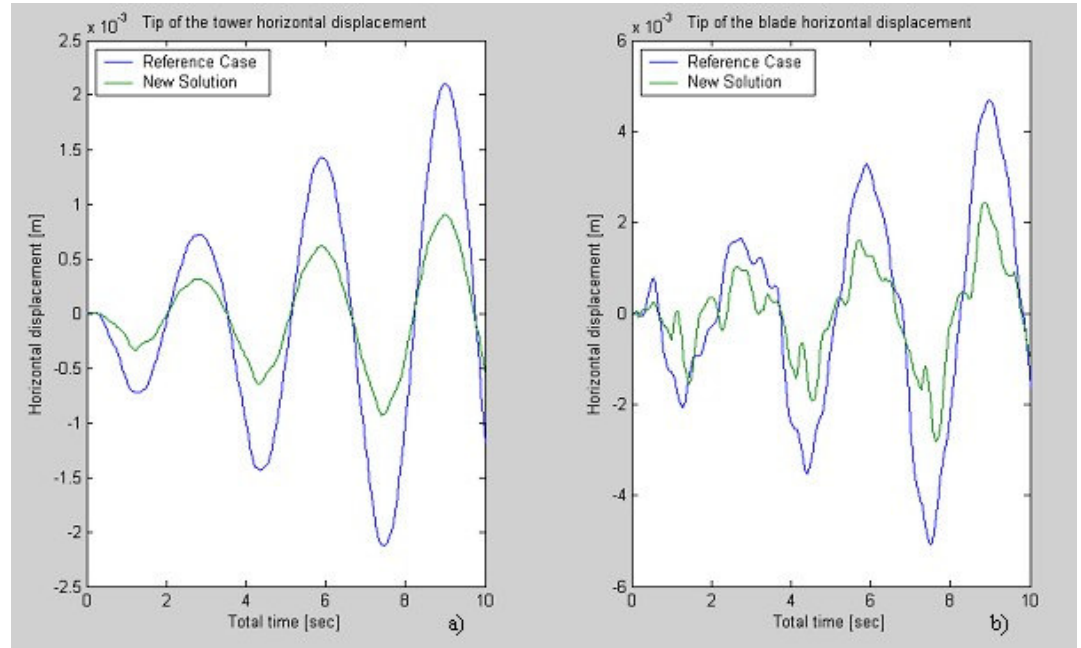

Figure 16: Tower (a) and blade (b) horizontal displacements

Applied period of dynamic ice loading resulted in resonant structural response. With blades' fundamental frequency being much higher than in case of tower, the response of blade consists of superposition of the tower response and response due to blades vibrations. Additional peaks in the response, visible in particular on the blade response for new solution case, come from the inertia effect of the moving cone. As the mass of cone is quite high compared to the mass of the whole structure, the inertia forces generated by the moving cone are big enough to influence the whole structure response.

Displacement results were sampled in equal time intervals and the distribution of results is shown on Figure 17.
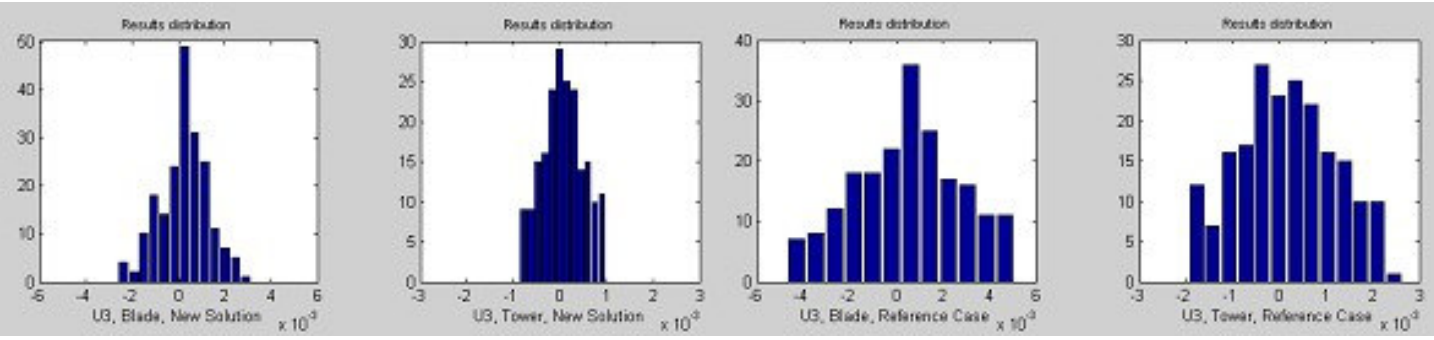

Figure 17: Blade and tower displacements - distribution of results 
In Table 3 root mean square values are compared along with maximum and minimum horizontal displacements. $41 \%$ decrease in horizontal reaction obtained in Simulation I corresponds to $45 \%$ and $48 \%$ decrease in the blade response, and $55 \%$ and $56 \%$ decrease in the tower response. The RMS values were reduced by 55\% and 58\% respectively. 58\% mitigation in the level of tower vibration can be especially useful from the fatigue strength point of view. Steel wind turbine towers are manufactured of tubular rings with welded flanges which are screwed together on site. Welds between flanges and steel body plates are subject to high fatigue and its mitigation would be highly appreciated.

\begin{tabular}{|l|c|ccc|c|c|}
\hline & RMS [m] & $\mathrm{U}_{\max }[\mathrm{m}]$ & $\mathrm{U}_{\min }[\mathrm{m}]$ & Change RMS & Change $\mathrm{U}_{\max }$ & Change $_{\text {min }}$ \\
\hline Blade, ref. case & 0.00229 & 0.00469 & -0.00510 & & \\
Blade, new sol. & 0.00103 & 0.00245 & -0.00281 & $55 \%$ & $48 \%$ & $45 \%$ \\
\hline Tower, ref. case & 0.00105 & 0.00210 & -0.00213 & $58 \%$ & $57 \%$ & $56 \%$ \\
\hline Tower, new sol. & 0.00044 & 0.00090 & -0.00093 & & & 5 \\
\hline
\end{tabular}

Table 3: Displacements comparison

\subsubsection{Cone behavior}

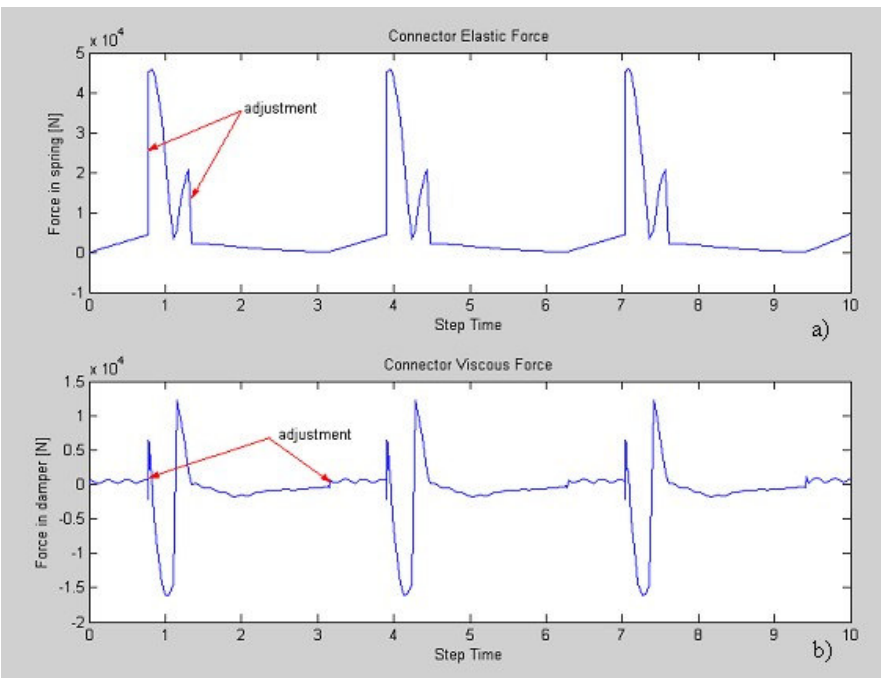

Figure 18: Connector force in spring (a) and damper (b)

The control scheme described in point 5.6.2 resulted in connector forces illustrated on Figure 18. The first graph shows the absolute value of elastic force in springs and the second graph depicts the viscous force in dampers. 
The first adjustment occurs at the same time for springs and dampers, and stands for changing parameters from their minimum to maximum values. The second adaptation occurs at different time instants for springs and dampers, and stands for changing the parameters back to their minimum (initial) values.

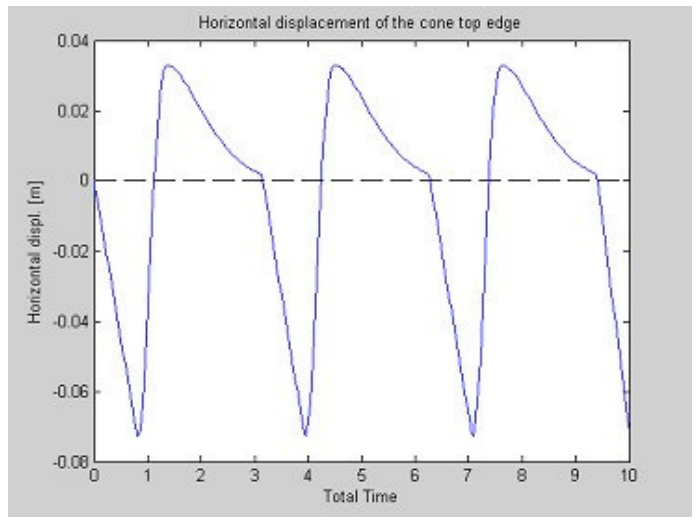

Figure 19: Cone top edge displacement

Time history data for horizontal displacement of the top edge of the cone is shown on Figure 19. It can be seen that the cone returns to its initial position before the next peak and thus can work in a repetitive sequence.

\section{CONCLUSIONS}

A new, semi-active solution aiming at mitigation of ice induced dynamic loading was introduced. Numerical feasibility study was performed in order to briefly assess the effectiveness of the new solution. Results seem to be promising, indicating about 50\% reduction in the structure's response. Due to high complexity of the ice modeling problem and lack of precise mechanical model of ice material, results need to be verified experimentally.

The effect of reaction force decrease due to the cone-tower connection compliance can be verified with a passive experimental set-up, where parameters $c$ and $k$ are constant. Interactions between cone behavior and ice sheet failure process could be investigated in such experiment in order to enhance the control procedure in further studies.

The effectiveness of present solution can be further increased by changing the position of axis of rotation along the tower shaft.

Finally, the cone inertia forces could be also used to influence the ice actions. For example, if the cone movement is not damped out before next contact with ice, then it could dynamically interact with the ice sheet, further enhancing its failure. Such effect could be used to prevent self induced excitation by changing the period of ice loading. This mode of the Smart Cone work could be switched on by the control device only during the danger of tower resonance. 


\section{ACKNOWLEDGMENTS}

The authors would like to acknowledge the financial support through the FP5 Research Training Networks Project HPRN-CT-2002-00284 (2002-2006) "SMART SYSTEMS - New Materials, Adaptive Systems and Their Nonlinearities: Modelling, Control and Numerical Simulation".

Present work was done at VTT Technical Research Centre of Finland as a result of researchers' exchange between IPPT, Warsaw, Poland and VTT, Espoo, Finland. The authors would like to gratefully aknowledge the support of VTT in acomplishing this work.

\section{REFERENCES}

[1] T.Karna and P.Jochmann, "Field Observations on Ice Failure Modes", Proc. 17 $7^{\text {th }}$ Int. Conf. Port and Ocean Eng. under Arctic Conditions, Trondheim, Norway (2003)

[2] A.Barker et al., "Ice loading on Danish wind turbines. Part 1: Dynamic model tests", Cold Regions Science and Technology 41(2005), p. 1-23.

[3] F.Li, Q.J. Yue, K.N. Shkhinek, T. Karna, "A Qualitative Analysis of Breaking Length of Sheet Ice Against Conical Structures", Proc. 17 $7^{\text {th }}$ Int. Conf. Port and Ocean Eng. under Arctic Conditions, Trondheim, Norway (2003).

[4] A.B. Cammaert and D.B. Muggeridge, Ice interaction with Offshore Structures, Van Nostrand Reinhold, New York (1988).

[5] T. Karna, K.Kolari, "Mitigation of Dynamic Ice Actions on Offshore Wind Turbines", Proc. $3^{\text {rd }}$ Eur. Conf. Structural Control, Vienna, Austria (2004).

[6] G. Lindqvist, and K. Juurmaa, "Suojausmenetelma ja sovitelma (A protection method and its application)", Finnish patent application Nr. 822157, (1982), published 28.09.1990.

[7] L.Y. Wang, "The oscillator-indenter for improving ice-induced vibrations", $6{ }^{\text {th }}$ Meeting of the ISO Working Group 8 for Arctic Offshore Structures, 10-12.12.2004. Presentation at the technical workshop of the meeting (2005).

[8] F.Li, Q.J. Yue, "An Analysis for Amplitude and Period of the Alternating Ice Load on Conical Structure", Proc. $16^{\text {th }}$ Int. Symposium on Ice, Dunedin, New Zealand (2002).

[9] Y.Qu, Q.J.Yue, X.Bi, T. Karna, "Random Ice Forces on Conical Structures", Proc. $17^{\text {th }}$ Int. Conf. Port and Ocean Eng. under Arctic Conditions, Trondheim, Norway (2003).

[10] J.F. Wilson, Dynamics of Offshore Structures, Wiley, New York (1984). 